Revista de Psicología de la PUCP. Vol. XX, 1, 2002

\title{
Estilos de afrontamiento y estatus performance en un grupo de pacientes oncológicos hospitalizados ${ }^{1}$
}

\author{
Cecilia Chau Pérez-Aranibar ${ }^{2}$ \\ Hugo Morales Córdova ${ }^{3} \quad$ Micaela Wetzell Espinoza ${ }^{4}$ \\ Pontificia Universidad Católica del Perú
}

\begin{abstract}
Se estudian las asociaciones entre estilos de afrontamiento medidos a través del Test de Estimación del Afrontamiento COPE-versión disposicional (Carver, Scheier y Weintraub, 1989) y Estado de Salud inferido a través del Estatus Performance, medido a través del Índice de Ejecución Conductual de Karnofsky, en un grupo de 28 pacientes oncológicos hospitalizados de ambos sexos. La investigación alcanza el nivel descriptivo-correlacional, y se encontraron asociaciones entre ambas variables expresadas en correlaciones Pearson positivas y moderadas interpretadas en el siguiente sentido: a mayor empleo del estilo, menor estatus de salud entre las escalas supresión de actividades competentes, postergación del afrontamiento, apoyo social instrumental, enfocar y liberar emociones y desentendimiento conductual. La escala afrontamiento activo, presentó una correlación negativa respecto al Estatus Performance como medida de salud.
\end{abstract}

Palabras clave: Estatus de salud, estatus performance, competencia inmunoglobulínica, estrés, afrontamiento, cáncer, calidad de vida, psiconcología, psicología de la salud, personalidad.

\section{Coping style and performance status in a group of oncological inpatients}

The associations between coping styles, measured by COPE Test, dispositional version (Carver, Scheier and Weintraub, 1989), and Health Status inferred through a performance status, measured by Karnofsky's Index of Behavioral Performance are examined. The study focuses upon 28 oncological inpatients. Positive moderate Pearson's correlations were found between these two variables in this correlational-descriptive study. These were interpreted in the following sense: the larger use of the style, the less health status among the scales suppression of competent activities, procrastination of coping, instrumental social support, focusing and releasing of emotions and behavioral disengagement. The active coping scale presented a negative correlation with regard to Performance Status as a health measure.

Key words: health status, performance status, immuneglobulinic competence, stress, coping, cancer, life quality, psychoncology, heath psychology, personality.

1 Se agradece la colaboración del Cuerpo Médico y la participación de los pacientes del Servicio de Oncología Médica del Hospital Nacional Edgardo Rebagliati Martins HNERM - EsSalud (Lima, Perú), así como la valiosa colaboración del Mag. Arturo Calderón en el análisis estadístico de los resultados del presente estudio.

2 Profesora Auxiliar de la Sección Psicología. Departamento de Humanidades de la PUCP.

3 Estudiante de pre-grado de la Especialidad de Psicología Clínica de la PUCP.

4 Estudiante de pre-grado de la Especialidad de Psicología de la PUCP. 

En las últimas décadas, los aportes de la Psicología de la salud en el campo de las enfermedades neoplásicas han cobrado especial importancia en tanto han favorecido la adaptación del paciente a su proceso de enfermedad y al tratamiento radiofarmacológico, la adaptación a los nuevos estilos de vida derivados del mencionado proceso, y atendido las especiales necesidades psicológicas generadas a partir del estado de enfermedad (Eysenck, 1995; Odgen, 1998; Taylor, 1991; Stein y Miller, 1993; Zeidner y Endler, 1996). En este sentido, el estudio permanente de las variables psicológicas con relación a los estados de salud, posibilitará esfuerzos concretos de intervención a un nivel preventivo, como el desarrollo de factores protectores hacia las enfermedades o el fortalecimiento de estilos de vida saludable.

La presente investigación explora las posibles relaciones entre los estilos de afrontamiento, entendidos como el conjunto de esfuerzos que las personas despliegan para enfrentarse a los problemas y dificultades encontradas en el ambiente y al estrés subsecuente que dichas situaciones generan, y el estatus performance en pacientes oncológicos hospitalizados.

\section{Afrontamiento, estrés y cáncer}

El estudio de la influencia de los factores psicológicos en el organismo se encuentra situado en un marco teórico amplio cuyo contenido principal es el conocimiento de las relaciones que se establecen entre las variables psicosociales y la enfermedad física (Stoudemire, 1995, en Cardenal, 1997a). Estudios en este campo adoptan un enfoque diferencial en el que pueden establecerse diferentes relaciones de influencia recíproca entre diversos tipos de variables psicológicas 
(rasgos de personalidad, estilos cognitivos, estilos de afrontamiento, entre otros), factores sociales (acontecimientos estresantes y/o sucesos vitales) y el inicio, desarrollo y progresión de variadas enfermedades físicas.

Las disciplinas que han iniciado estos estudios han sido, tradicionalmente la Psicología y la Medicina, a través de la Medicina psicosomática, que ha sido útil en tanto ha enlazado las diferentes teorías procedentes de ambas ciencias. En un principio la Medicina psicosomática tenía un marcado carácter psicógeno en la consideración etiológica del trastomo, sugiriendo que todas las anomalías físicas tenían causas psicológicas, frente a las orientaciones médicas biologicistas que proponían a las patologías anátomo funcionales como causas preponderantes de las enfermedades.

En la actualidad, renovadas orientaciones dentro de la Psicosomática consideran ambos factores, la dinámica psicológica y la disposición genética, como decisorios en el origen y progreso de las enfermedades, además de reconocer la compleja naturaleza multicausal de las mismas. No obstante, la necesidad de una integración interdisciplinar de las orientaciones más relevantes de la Psicología y de sus métodos de investigación para una comprensión holística del ser humano y de los procesos de salud y enfermedad demandará esfuerzos aún insuficientes, sea desde los aportes de la Medicina conductual o de la Psicología de la salud desde una perspectiva conductual-cognitiva.

Precisamente, desde la perspectiva conductual-cognitiva de la Psicología de la salud, se ha abordado el estudio de la influencia de una variable psicológica conocida como afrontamiento (del inglés coping, propuesto como categoría de análisis en el estudio del fenómeno del estrés dentro de la teoría Transaccional del estrés de Lazarus y Folkman, 1986) en la evolución de enfermedades como el cáncer, los trastornos cardiovasculares, la diabetes y el SIDA (Cardenal, 1997b). 
Lazarus define el afrontamiento como el proceso por el cual un individuo se esfuerza cognitiva y conductualmente para enfrentar una situación considerada estresante para después ejecutar una acción (Lazarus, 1991). Su función principal es reducir la tensión y devolver el equilibrio (la homeostasis general) al organismo. Así mismo, puede ser situacional o disposicional. En la modalidad situacional, se plantea que el afrontamiento es un proceso que cambia a través del tiempo y por las circunstancias, es decir es un proceso dinámico (Lazarus y Folkman, 1986); mientras que en la segunda modalidad, se señala que el afrontamiento es un estilo personal relativamente estable, es decir no cambia, y ambos están íntimamente relacionados (Carver y Scheier, 1994). Según Lazarus y Folkman (1986), el afrontamiento está enfocado tanto hacia el problema como hacia la emoción. En el primer caso, se refiere a que el individuo procederá a definir el problema y buscará alternativas para solucionarlo y de esta manera modificará la realidad o situación en la que se encuentra, y en el segundo caso, se refiere a la disminución del trastorno emocional. Adicionalmente, se ha propuesto la existencia de otro tipo de afrontamiento enfocado hacia la percepción o evitativo (Contrada, Leventhal y O’Leary, 1990; Cooper y Payne, 1991 y Flores, 1999).

Los estilos de afrontamiento se refieren al conjunto de técnicas o métodos que el individuo elabora en respuesta a una situación estresante considerada desbordante. Carver, Scheier y Weintraub (1989) proponen 13 estilos:

Estilos de afrontamiento enfocados en el problema. Comprenden al afrontamiento activo, es decir, actuar con el fin de solucionar el problema; la planificación -cómo elaborar una estrategia-; la supresión de actividades competentes, es decir, dejar de hacer otras actividades con el fin de concentrarse en el problema; la postergación del afrontamiento -cómo esperar el momento adecuado para actuar-; y la búsqueda de apoyo social por motivos instrumentales, es decir, buscar el consejo de los demás, alguna opinión o información relevante, etc. 
Estilos de afrontamiento enfocados en la emoción. Comprenden la búsqueda de apoyo social por motivos emocionales, es decir, buscar apoyo moral; reinterpretación positiva y crecimiento, es decir, ver el lado positivo o bueno del problema; aceptación, como aceptar y asumir el problema; acudir a la religión; y negación, es decir, negar que exista el problema.

En cuanto a los otros estilos o estilos evitativos (también conocidos como afrontamiento enfocado en la percepción) se observó: enfocar y liberar emociones, es decir, liberar las emociones de manera abierta; desentendimiento conductual -como dejar de actuar a fin de solucionar el problema-; y desentendimiento mental, es decir, tratar de distraerse para no pensar en el problema (Flores, 1999). Por otro lado, Carver y Scheier en 1994 (Rojas, 1997) identificaron dos nuevas categorías de afrontamiento, éstas son: uso de alcohol y drogas, y recurrir al humor.

El estrés, núcleo de activación del afrontamiento, se define como la relación entre el individuo y el medio, el cual es evaluado como amenazante o desbordante de nuestros recursos (Lazarus y Folkman, 1986). Así mismo, éste incluye tanto las exigencias del medio o "estresores", como las reacciones de una persona ante ellas o las "respuestas con estrés" a las mismas (Darley, 1990; en Cella, Orofiamma y Holland, 1987). Los estresores se generan por los cambios mayores que afectan a grandes masas como los desastres naturales, o los producidos por el hombre; los cambios mayores que afectan a una persona o pocas como la muerte de un ser querido, amenaza a la propia vida, enfermedad incapacitante, pérdida del trabajo, divorcio, dar a luz a un recién nacido, dar un examen importante; o las demandas cotidianas como tener que hacer muchas tareas, lidiar con el tráfico, atender a los hijos pequeños, etc. Así mismo, las reacciones al estrés pueden ser fisiológicas, emocionales y cognitivas (Lazarus, 1982, en Breznitz y Goldberger, 1993). 
Estilos de afrontamiento y estatus perfomance en un grupo de pacientes

En cuanto a las primeras, hallazgos recientes (Stein y Miller, 1993) indican una proliferación de los linfocitos T-4 y muerte de células que han experimentado estrés, existiendo conexión entre la duración del estrés y la magnitud del cambio fisiológico. Además, se producen cambios en el número de células blancas y la cantidad de anticuerpos, ya que la habilidad del cuerpo para producirlos está relacionada con el nivel de ansiedad que se experimenta a causa del estrés, y por tanto se observa que a mayor ansiedad, se producen menos anticuerpos. Las segundas son principalmente temor, excitación, cólera, depresión, miedo, ira, etc.; y las terceras pueden ser preocupación, pérdida de control y negación, las cuales pueden ir acompañadas de bloqueos mentales, pérdida de la memoria, sensación de irrealidad y procesos disociativos de la mente (Valdés y De Flores, 1990).

Las demandas ambientales producen estrés, pero la manera como se interpreta y reacciona ante éstas depende de cada individuo, ya que las personas difieren en cuanto a su sensibilidad y vulnerabilidad hacia las mismas. Es por esta razón que cada persona ante una situación considerada estresante tiene una determinada evaluación cognitiva la cual está considerada como un proceso que determina las consecuencias que un acontecimiento o demanda estresante provocará en el individuo (Lazarus y Folkman, 1986).

La evaluación puede ser primaria y/o secundaria. En la primera, se evalúa la relación entre el individuo y el medio ambiente o sus procesos internos según la importancia que él le otorgue, y en la segunda se evalúan los recursos que el individuo posee para afrontar una situación estresante. Una tercera y menos frecuente etapa dentro de la evaluación, es la reevaluación, la cual consiste en una retroalimentación que informa sobre los cambios y permite reemplazar las valoraciones iniciales (hechas en la primera etapa de la evaluación) por otras nuevas (Labrador y Crespo, 1994).

En este sentido autores como Eysenck y Grossarth-Maticek (en Eysenck, 1995) intentan demostrar que diferentes tipos de reacción 
ante el estrés pueden asociarse consistentemente a determinados trastornos físicos. Sheier y Bridges (en Bleiker, Van der Ploeg y Arder, 1995), realizaron un estudio de la evidencia prospectiva de ciertas variables de personalidad que ejercen algún efecto en el desarrollo y desenlace de alguna enfermedad física. La revisión considera el efecto de variables como hostilidad y enfado, supresión emocional, depresión, fatalismo y pesimismo sobre enfermedades coronarias, cáncer y SIDA. Ambos presentan un modelo que integra todas estas variables en un esquema conceptual global. Además, identifican distintas variables que ejercen cierto efecto moderador sobre la relación que existe entre ciertos aspectos de la personalidad y la salud.

En el estudio sobre las relaciones entre variables psicológicas (como el afrontamiento) y cáncer, algunos investigadores se han centrado en buscar meras asociaciones descriptivas entre ciertos rasgos de personalidad y el padecimiento del cáncer. Otro grupo de investigadores ha establecido la existencia de relaciones entre ciertas emociones y la función inmune. En este sentido, Levenson y Bemis en 1995 (en Odgen, 1998) analizan los resultados de distintos estudios que investigan dos hipótesis. La primera plantea que ciertas variables psicosociales afectan el padecimiento y progresión del cáncer. La segunda hipótesis planteada estudia la posibilidad de que ciertos factores psicológicos afecten al sistema inmune, que a la vez puede contribuir al padecimiento y progresión del cáncer.

Las variables psicosociales examinadas incluyen estados afectivos, estilos de afrontamiento, rasgos de personalidad y eventos estresantes. Los sistemas orgánicos que sirven de conexión entre variables psicológicas y enfermedad son fundamentalmente: sistema nervioso central y autónomo, sistema neuroendocrino, sistema de péptidos y sistema inmune; sistemas cuya interacción en conjunto constituyen la red psiconeuroinmunoendocrina.

Cardenal (1997b), identifica en sus investigaciones el estilo de afrontamiento evitativo o de evitación emocional como frecuente- 
Estilos de afrontamiento y estatus perfomance en un grupo de pacientes

mente empleado entre los pacientes oncológicos. Otras contribuciones exploran las implicancias de un estilo de personalidad represora sobre el organismo y la salud, estudiando su relación con el estrés y sus efectos en el sistema inmune, en enfermedades como el cáncer y la artritis reumatoide.

Las estrategias de afrontamiento que entran dentro de la clasificación general de orientadas hacia el problema o de abordaje activo, suelen ir asociadas a un menor número de síntomas físicos. De forma inversa, las orientadas hacia la evitación y el manejo de las emociones, se relacionan en general a un mayor número de afecciones físicas. Dentro de los estudios que han investigado la relación entre el afrontamiento y el desarrollo del cáncer se encuentran: mejores resultados clínicos asociados al afrontamiento activo y al afrontamiento orientado hacia el problema, ocurriendo lo contrario en el uso de estrategias de evitación, concretamente la negación y el afrontamiento represivo. Personas resistentes al estrés utilizan mucho menos la evitación como estrategia de afrontamiento, en comparación con personas afectadas por el estrés.

El uso de la evitación y la negación se ha asociado con un detrimento de variables inmunes en personas seropositivas, con una progresión más rápida del SIDA en un lapso de más de siete años y con un mayor avance de la enfermedad (Kiecolt-Glaser y Glaser, 1986). Contrariamente a estos resultados, otros estudios indican que la negación o la evitación pueden asociarse positivamente con parámetros de salud. Por ejemplo, se ha encontrado que la aceptación realista disminuye la supervivencia entre individuos con SIDA, y que la evitación puede ir asociada a una menor progresión de la infección de HIV-1 (Schneiderman, Antoni, Saab y Ironson, 2001).

Uno de los estudios prospectivos-longitudinales más importantes realizados en España sobre afrontamiento y cáncer (Eysenck, 1995) examina la relación entre la autorregulación, evaluada mediante un 
test de personalidad y salud. Un total de 5,716 personas fueron evaluadas y se les realizó un seguimiento durante 15 años para demostrar la alta predictibilidad de la mortalidad en trastornos oncológicos. Para estudiar el papel que desempeñan los factores de riesgo, se diseñó especialmente una escala que evaluaba el conocimiento que se tenía sobre los siguientes factores de riesgo: genéticos, ejercicio, nutrición, alcohol y tabaco. Se administró a un grupo de 3,240 personas el Personality Stress Inventory y el Grossarth-Maticek Typology (Grossarth-Maticek y Eysenck, 1990).

Se encontró que de los seis (6) tipos de reacción ante el estrés que establecen Grossarth-Maticek y Eysenck, los tipos 1 y 5 aparecían frecuentemente asociados al padecimiento del cáncer. El tipo 1 se caracteriza por presentar elevado grado de dependencia conformista respecto a algún objeto o persona con valor emocional destacado para la persona, e inhibición para establecer intimidad o proximidad con las personas queridas. Son individuos que ante situaciones estresantes suelen reaccionar con sentimientos de desesperanza, indefensión y tendencia a reprimir las reacciones emocionales abiertas. La pérdida o ausencia del objeto se mantiene como fuente de estrés, ya que la persona no se desvincula definitivamente de él, pero tampoco mantiene la proximidad e intimidad necesaria.

El tipo 5 o racional-antiemocional, presenta una tendencia a emitir reacciones racionales y antiemocionales. Este tipo de personas suele suprimir o negar las manifestaciones afectivas, encontrando dificultad para expresar sus emociones. Suelen encontrar predisposición a la depresión y al cáncer, así como a la artritis reumatoide. Entre ellas se observa un predominio de lo racional sobre lo emocional. Las personas de este tipo muestran una tendencia a aplicar defensas como la racionalización y la intelectualización y no expresan sus sentimientos, especialmente aquellos considerados por otras personas como negativos tales como la hostilidad y la ira. En ocasiones estas personas son altamente escrupulosas y precisas, y su comportamiento puede ser calificado como compulsivo. 
La clave sobre los mecanismos que hay que tener en cuenta para relacionar factores psicológicos como el afrontamiento y enfermedades físicas como el cáncer, se encuentra en las investigaciones sobre estrés y sistema inmunitario en animales (Rosenzweig y Leiman, 1993). Se ha observado que varios tipos de estrés producen aumento en los niveles circulantes de hormonas adrenales, especialmente corticoesteroides. Se sabe que la corticoesterona tiene sobre el sistema inmune potentes efectos supresores, entre los que se hallan disminución del número de linfocitos circulantes, reducción del timo y cierta pérdida de tejido de los ganglios linfáticos. Por tanto, la efectividad del sistema inmune se reduce en los estados de estrés.

Riley (Rosenzweig y Leiman, 1993) ha sugerido que el estrés aumenta la vulnerabilidad a agentes que causan cáncer como los virus. Algunos experimentos de este investigador apoyan esta idea. En un estudio experimental, este autor estresaba a ratones con tumores implantados haciéndoles soportar un breve período de rotación corporal forzada. A continuación de este tratamiento los tumores incrementaron su tamaño hasta ser cuatro veces mayores que los animales de control. Un efecto similar se produce con la administración directa de corticoesterona sin experiencia de estrés, hallazgo que apoya la idea de que la liberación de hormonas de la corteza adrenal activada por el estrés puede incrementar la susceptibilidad al cáncer o modificar su progresión.

Desde el punto de vista de la evaluación cognitiva y el afrontamiento en relación con las emociones y sus relaciones con el sistema inmune, observamos que las emociones experimentadas como positivas serán producidas por la evaluación de una situación como beneficiosa; mientras que las emociones experimentadas como negativas, por la valoración de una situación como dañina, de pérdida o de amenaza (Valdez, 1999). Para contrarrestar el malestar producido por una emoción desagradable las personas cuentan con diversos mecanismos de control emocional. Así, las personas pueden emplear estilos de afrontamiento que cambien la situación que provoca la emoción (afronta- 
miento enfocado al problema), o intentar reducir la intensidad de dicha reacción emocional (afrontamiento enfocado a la emoción). Estos estilos de afrontamiento han sido ya descritos en líneas anteriores, y se sabe que existen otras clasificaciones y tipos de afrontamiento (Rodríguez, 1995; Simón, 1993).

Weinberger (1990, en Buendía, 1993a) ha tipificado como estilo represivo de afrontamiento, aquel estilo por el cual la persona conserva y retiene en sí misma el displacer emocional producido por un estímulo externo o interno, al cual no se enfrenta directamente, sino por el contrario, tolera y contempla pasivamente, inhibiendo por completo la expresión abierta del mismo, constituyéndose en un patrón de afrontamiento mal adaptativo y patógeno por su uso en exceso (Buendía, 1993b). Los antecedentes de dicho estilo de reacción ante el estrés, se remontan a la formulación inicial de Freud en 1915 sobre las defensas inconscientes (Carlson y Hatfield, 1992), en las cuales, la represión ejercida sobre la memoria de sucesos específicos en la cognición consciente, reprime el recuerdo de los sucesos teñidos con emociones desagradables, con la finalidad de no experimentarlas y así no alterar el equilibrio del sistema conciente y sus transacciones con la realidad (represión entendida como inhibición de la capacidad para experimentar emociones).

El control o represión de experiencias desagradables para eliminar un malestar importante, puede tener consecuencias peligrosas para el individuo. Existen algunos datos de investigaciones que señalan que los individuos con un estilo represivo de afrontamiento no aceptan el diagnóstico de cáncer (además de emplear la negación como estilo de afrontamiento), no siguen las prescripciones médicas, reciben dosis menores de quimioterapia, y esto puede afectar negativamente su esperanza de vida (Bonadonna, 1981). Los individuos represores de emociones negativas quedan definidos en la literatura psicológica como aquellos que presentan bajas puntuaciones en ansiedad y altas puntuaciones en deseabilidad social, evaluadas a través de cuestionarios de autoinforme, y a la vez, altos índices de ansiedad evaluados a través de registros de medida de la respuesta fisiológica. 
Estilos de afrontamiento y estatus perfomance en un grupo de pacientes

El individuo represor intenta controlar todas las respuestas que componen una emoción negativa; sin embargo el control voluntario que posee sobre cada una es diferente, consiguiendo así reducir unas respuestas pero no otras, lo que originará una alta discordancia entre los tres sistemas de respuesta emocional (conductual, fisiológico y cognitivo). Se considera por tanto que el sujeto mostrará una alta activación fisiológica pero podrá controlar bien la experiencia emocional y la expresión abierta y observable de su reacción emocional.

En relación con el cáncer, en multitud de estudios se ha encontrado que los individuos con cáncer presentan un patrón de estilo represivo de afrontamiento. Algunos autores consideran que es la inhabilidad para expresar emociones (especialmente ansiedad e ira), o el control emocional; el indicador más característico entre las personas que padecen de cáncer, intentando relacionar este hecho como progresión del mismo, mientras que otros autores lo cuestionan apelando a la heterogeneidad de los diferentes tipos de cánceres estudiados y la evidencia de predisposición genética a padecer de ciertos tipos de cáncer (Kiecolt-Glaser y Glaser, 1986; Kiecolt-Glaser, McGuire, Robles y Glaser, 2002).

Por otro lado, Cano y Vindel (1994, en Cardenal, 1997b) encontraron que los individuos con distintos tipos de cáncer comparados con un grupo control de individuos sanos equiparados en edad, sexo y nivel cultural, presentaban como grupo un patrón típico de potencial estilo represivo de afrontamiento, con baja ansiedad y alta deseabilidad social, resultando ser más altos en rasgo de control emocional, en control de la ansiedad ante las revisiones médicas, más bajos en la expresión de ira, y más altos en racionalización y antiemocionalidad. En estudios más recientes (Cano-Vindel y Miguel-Tobal, 1996, en Cardenal, 1997b) se ha encontrado que existe una mayor incidencia de un potencial estilo represivo de respuesta en un grupo de mujeres con cáncer de mama al ser comparadas con un grupo control de mujeres sanas equivalentes en edad y otro tipo de variables socio-demográficas. 
Parece ser, por tanto, que existe una mayor incidencia de individuos represores de emociones en el grupo de personas con cáncer que en grupos control, y dentro del grupo de individuos con cáncer, los represores son los que tienen peor pronóstico en su enfermedad. Aunque el estilo represivo ha sido asociado con un mal funcionamiento del sistema inmune, existe un problema metodológico importante a la hora de determinar si existe algún tipo de relación causal entre represión de emociones y cáncer, ya que el estilo de afrontamiento podría ser muy bien una consecuencia del diagnóstico de cáncer, más que un factor potencialmente cancerígeno. Sin embargo al referirnos a un afrontamiento de naturaleza disposicional, asumimos que el estilo de afrontamiento es un factor interno (constitutivo de la personalidad) que favorece la aparición de las neoplasias malignas.

En este sentido, no parece apropiado establecer que el estilo represivo de afrontamiento sea causante del cáncer, aunque sí un buen predictor de dicha enfermedad. De este modo, debemos adoptar una aproximación multicausal de la etiopatogenia del cáncer, teniendo en cuenta que la importancia de los factores psicológicos será distinta para los diferentes tipos de cáncer. En la actualidad, la mayoría de las investigaciones evalúan el estilo de afrontamiento represivo principalmente a través de medidas de autoinforme, prescindiendo del componente fisiológico.

Sin embargo, como ya se ha mencionado anteriormente, en estudios en los que se emplean registros de medidas fisiológicas, se reporta que un elevado control racional de emociones tiene un impacto negativo sobre el funcionamiento del aparato inmune. En consecuencia sería importante incorporar a los estudios sobre los estilos de afrontamiento en pacientes oncológicos medidas psicofisiológicas y en especial relacionadas con el funcionamiento del sistema inmune, dada la importancia que presenta en el transcurso de los tratamientos. En ese sentido algunos autores recomiendan el estudio de variables hematológicas ligadas a la competencia inmunológica e inmunoglo- 
bulínica, en especial monocitos y neutrófilos en el primer caso, y nivel de citokinas e interleukinas 2 y 6 en el segundo caso, como indicadores de la respuesta fisiológica a las situaciones de amenaza, por considerarse variables simples, rápidas y fáciles de obtener.

En la presente investigación, el estilo de afrontamiento represivo será equivalente -dentro de la teoría Transaccional del estrés de Lazarus y Folkman (1986) y de acuerdo a la clasificación de Flores (1999)a dos estilos de afrontamiento enfocados en la emoción: negación y aceptación, mientras que el estilo de afrontamiento evitativo a las tres escalas adicionales incluidas en el COPE (Carver, et al., 1989), conocidas como estilos evitativos de afrontamiento: enfocar y liberar emociones, desentendimiento conductual y desentendimiento mental.

\section{Planteamiento del Problema}

El presente estudio tiene como objetivos principales identificar los estilos de afrontamiento y el estatus performance que presentan los pacientes oncológicos hospitalizados, y determinar las posibles asociaciones (medidas a través del coeficiente de correlación de Pearson) entre ambas variables en la muestra de estudio.

En ese sentido, se plantea la siguiente pregunta de Investigación: ¿Existen relaciones entre los estilos de afrontamiento empleados por los pacientes oncológicos hospitalizados de la muestra en estudio y el estatus performance de los mismos?

A continuación se describen las variables empleadas:

- Afrontamiento.- Según Lazarus y Folkman (1986), comprende todos aquellos esfuerzos dinámicos, cognitivos y conductuales que despliega el individuo para enfrentar el estrés, lo que representa, manejar tanto las demandas específicas internas como externas, que son evaluadas como excedentes o desbordantes de los recursos del individuo. 
Según la clasificación de Lazarus y Folkman (1986), existen dos tipos de dirección u orientación del afrontamiento:

Focalizado en el Problema. Se manifiesta cuando el individuo busca modificar la relación existente, sea alejando o disminuyendo el impacto de la amenaza y comprende las siguientes escalas:

Escala 1: Afrontamiento Activo. Se define como el conjunto de realizar conductas orientadas a incrementar los esfuerzos con el fin de distanciar o evitar el estresor, o minimizar sus efectos negativos.

Escala 2: Planificación. Proceso consistente en pensar cómo manejarse frente al estresor organizando y planificando estrategias de acción, premeditando qué escoger y estableciendo cuál será la mejor estrategia para manejar el problema.

Escala 3: Supresión de actividades competentes. Representa el pasar por alto o subestimar otras posibles actividades evitando ser distraído, con el objeto de manejar mejor el estresor.

Escala 4: Postergación del afrontamiento. Se define como la capacidad de esperar o postergar el afronte del estresor hasta que se presente la oportunidad apropiada para actuar sobre el problema, impidiendo realizar alguna acción prematuramente.

Escala 5: Búsqueda de apoyo social por razones instrumentales. Significa buscar en las otras personas consejo, asistencia e información u orientaciones con el fin de solucionar el problema.

Focalizado en la Emoción. Se dirige a disminuir o eliminar las reacciones emocionales desencadenadas por la fuente estresante. Comprende las siguientes escalas:

Escala 6: Búsqueda de apoyo social por razones emocionales. Consiste en la búsqueda de apoyo moral, empatía y comprensión en las demás personas, con la finalidad de disminuir la reacción emocional negativa.

Escala 7: Reinterpretación positiva y crecimiento. Estilo en e1 que el individuo evalúa el problema de manera positiva, reconociendo o atribuyendo cualidades favorables a la experiencia estresante en 
sí misma, es decir, rescatando lo favorable de la situación adversa, y asumiéndola como un aprendizaje para el futuro.

Escala 8: La aceptación. Comprende. dos aspectos durante el proceso de afrontamiento. El primero consiste aceptar que la situación estresante realmente existe y acontece durante la fase de evaluación primaria. El segundo tiene lugar durante la evaluación secundaria y consiste en aceptar la realidad como un hecho con el que se tendrá que convivir ya que es inmodificable en el tiempo.

Escala 9: Negación. Se define como rehusarse a creer que el estresor existe, o comportarse y pensar como si el estresor fuera irreal o sencillamente no existiese.

Escala 10: Acudir a la religión. Tendencia a apoyarse en la religión en situaciones de estrés con la intencionalidad de reducir la tensión existente.

Las escalas de afrontamiento adicionales u otras escalas de afrontamiento, encontradas por Carver y colaboradores son (Carver, et al., 1989):

Escala 11: Enfocar y liberar emocione.s Ocurre cuando el individuo se focaliza en las consecuencias emocionales desagradables o displacenteras que se experimentan expresando abiertamente dichas emociones.

Escala 12: Desentendimiento conductual. Consiste en la disminución del esfuerzo conductual y cognitivo dirigido al estresor, dándose el individuo por vencido sin intentar alcanzar las metas con las que el estresor está interfiriendo.

Escala 13: Desentendimiento mental. Ocurre cuando el individuo permite que una amplia diversidad de actividades lo distraigan de su evaluación de los aspectos conductuales del problema, o de la meta con la que el estresor esta interfiriendo.

Esta variable es medida por los puntajes obtenidos en el cuestionario de estimación del afrontamiento (COPE) en la muestra estudiada. 
- Estatus Performance. - Es el estado o condición físico-orgánica favorable en el paciente para la ejecución de conductas abiertas emitidas conscientemente, desde realizar alguna actividad física sencilla como ocuparse de las propias necesidades (desplazarse, subir escaleras, levantar objetos, etc.) y trabajar hasta el fallecimiento del paciente que supone un nivel de ejecución conductual nula.

Esta variable es medida por los puntajes obtenidos tras la aplicación del Índice del Estado de Ejecución Conductual de Karnofsky (OMS, 1995) en la muestra estudiada.

Para efectos de la obtención del coeficiente de correlación de Pearson entre las variables en estudio, se empleó la equivalencia de los niveles de ejecución conductual descritos en el Índice de Ejecución Conductual de Karnofsky con los niveles del ZUBROD (ambos en escala de razón y de compatible medición e interpretación).

\section{Cuadro 1}

Equivalencia entre las escalas de estatus performance o de ejecución conductual

\begin{tabular}{|c|c|c|c|c|}
\hline \multicolumn{5}{|c|}{ KPS/Zubrod"-Ecog-Who-Oscb (S. Carlo Borromeo Hospital) } \\
\hline $100=0$ & $80-90=1$ & $60-70=2$ & $40-50=3$ & $20-30=4$ \\
\hline
\end{tabular}

Nota. "Escalas empleadas en el presente estudio.

\section{Metodología}

De acuerdo a Hernández, Fernández y Baptista (1998), el nivel de Investigación alcanzado en el presente estudio correspondió al nivel Descriptivo-Correlacional. Descriptivo porque mide los estilos de afrontamiento y el estatus performance, y correlacional porque mide el grado de asociación entre ambas variables. El tipo de investigación optado fue no experimental, ya que no se manipularon variables independientes (Kerlinger, 1988). 
Estilos de afrontamiento y estatus perfomance en un grupo de pacientes

\section{Participantes}

En la presente investigación participaron 28 pacientes oncológicos hospitalizados entre varones y mujeres, de nivel socioeconómico preponderantemente medio-bajo (también de niveles alto-medio, medio y bajo), católicos cristianos (a excepción de un participante evangélico y otro universalista) con un rango de edad comprendido entre los 14 y 68 años, del Servicio de Oncología Médica del Hospital Nacional Edgardo Rebagliati Martins-EsSalud. La edad promedio entre los participantes fue de 42 años (41.85), distribuidos entre 14 mujeres y 14 varones, con el siguiente nivel de instrucción: 11 participantes con un nivel de educación superior universitaria, 5 participantes con educación superior técnica y 11 participantes con educación secundaria completa, mientras que sólo 1 participante contaba con educación primaria completa.

Respecto al diagnóstico clínico en la muestra, se observaron los siguientes diagnósticos agrupados en las siguientes frecuencias: 5 pacientes con cáncer gástrico, 5 pacientes con linfoma no Hodgkin, 5 pacientes con cáncer de mama, 3 pacientes con cáncer testicular, 2 pacientes con cáncer cervical, 2 pacientes con linfoma no especificado, 1 paciente con leucemia linfática aguda, 1 paciente con cáncer epidermoide, 1 paciente con cáncer al páncreas, 1 paciente con fibrohistiocitoma y 2 pacientes con presunción diagnóstica de cáncer pulmonar y cáncer gástrico respectivamente. El tiempo promedio de comunicación del diagnóstico es de tres años. Así mismo, 26 participantes declaran haber recibido, hasta el inicio del estudio, tratamiento quimioterapéutico y radioterapia, a excepción de los dos pacientes varones con presunción diagnóstica de cáncer pulmonar y cáncer gástrico.

Para fines del presente estudio, se optó por el muestreo de tipo no probabilístico intencional, ya que no se dispuso de los recursos necesarios para obtener una base de datos que comprenda a la población en estudio. Se realizó el estudio con 28 participantes debido a las dificultades encontradas durante el desarrollo del mismo, en términos de muerte experimental, disponibilidad personal por causas de salud (tra- 
tamiento de quimioterapia) y razones personales (participantes que aceptaron colaborar en un principio pero después desistieron).

\section{Instrumentos}

Cuestionario de Estilos de Afrontamiento (COPE)

Se utilizó el instrumento COPE (Coping Estimation) o Estimación del Afrontamiento, desarrollado por Carver, Scheier y Weintraub (1989) en Estados Unidos. Fue construida basándose en dos modelos teóricos: el de Richard Lazarus y el modelo de autorregulación conductual de Bandura. Según los autores, esta escala pretende abarcar áreas de afrontamiento que no se consideraron en el Inventario de Tipos de Afrontamiento desarrollado por Lazarus y Folkman en 1980. Este instrumento fue adaptado por Casuso (1996; en Chau, 1998) en población universitaria limeña.

Los autores de la prueba validaron este instrumento a través de la validez de constructo, para ello sometieron las respuestas obtenidas a un análisis factorial de rotación oblicua obteniendo 12 factores con eigenvalues mayores a 1.0, de los cuales 11 fueron fácilmente interpretados y los otros no llegaron a una carga factorial de .30 , por lo que no se incluyó en el resultado final. La composición de los 11 factores estuvo acorde con la asignación de elementos a las escalas; a excepción de dos escalas: el afrontamiento activo y la planificación, que aparecieron juntos como un solo factor. Sin embargo, se consideró más adecuado asignarlos como dos factores independientes, siguiendo los criterios teóricos sobre el constructo. Lo mismo sucedió con las escalas: búsqueda de soporte social, donde se procedió de la misma forma.

Se obtuvo la validez ítem/test de las escalas del COPE a través del análisis de correlación ítem/test, el cual exigió una correlación superior a $r=.20$ para que el ítem fuese aceptado como válido y discriminativo (Kline, 1993). Posteriormente se obtuvo la mediana de la correlación ítem/test. La confiabilidad de las mediciones por escala, se obtuvo a 
Estilos de afrontamiento y estatus perfomance en un grupo de pacientes

través del coeficiente de consistencia interna alfa de Cronbach, que se consideró significativo cuando era igual o mayor a .60 .

El COPE está constituido por 52 ítems en formato tipo Likert de 4 puntos de calificación (del 1 al 4), distribuidos en tres áreas comprendidas en 13 escalas que corresponden a los trece estilos de afrontamiento. Cada una de estas escalas está conformada por 4 ítems, los cuales se califican de acuerdo a una plantilla. Los puntajes obtenidos en cada ítem se suman con sus correspondientes en cada escala, de tal forma que se obtienen 13 puntuaciones acumuladas que permiten identificar un perfil de afrontamiento. Finalmente, se establece una jerarquía que permite determinar qué estilos de afrontamiento son los más utilizados.

\section{Cuadro 2}

Estilos de afrontamiento en el COPE en la clasificación de Carver, Scheier, y Weintraub (1989)

\begin{tabular}{|llc|}
\hline Estilos & \multicolumn{1}{c|}{ Coping Estimation (COPE) } & \multicolumn{1}{c|}{ Ítems } \\
\hline Estilo 1 & Estilo de afrontamiento enfocado en el problema & \\
& Afrontamiento activo & $1,14,27$ y 40 \\
& Planificación & $2,15,28$ y 41 \\
& Supresión de actividades competentes & $3,16,29$ y 42 \\
& Postergación del afrontamiento & $4,17,30$ y 43 \\
& Búsqueda de apoyo social por motivos instrumentales & $5,18,31$ y 44 \\
& & \\
Estilo 2 & Estilos de afrontamiento enfocado en la emoción & \\
& Búsqueda de apoyo social por motivos emocionales & $6,19,32$ y 45 \\
& Reinterpretación positiva y crecimiento & $7,20,33$ y 46 \\
& Aceptación & $8,21,34$ y 47 \\
& Acudir a la religión & $9,22,35$ y 48 \\
& Negación & $11,24,37$ y 50 \\
Estilo 3 & Escalas adicionales propuestas por Carver & $10,23,36$ y 49 \\
& Enfocar y liberar emociones & $12,25,38$ y 51 \\
& Desentendimiento conductual & $13,26,39$ y 52 \\
\hline
\end{tabular}


Cecilia Chau Pérez-Aranibar, Hugo Morales Córdova, Micaela Wetzell Espinoza

\section{Índice del Estado de Ejecución Conductual de Karnofsky}

Se empleó la Escala Internacional de medición del estado físico para la ejecución conductual de Karnofsky como medida indirecta del estado de salud inferida a partir de la ejecución conductual.

Las Escalas de medición del Estatus Performance como medidas indirectas del estatus de salud en pacientes oncológicos fueron diseñadas y validadas por la Organización Mundial de la Salud a fines de 1943 (De Vita, 2000). Los valores numéricos (numerales) consignados en estas escalas van desde la ausencia de sintomatología cancerígena y colateral -en este sentido la presencia de un estatus de salud más alto- (representadas en el ZUBROD en el nivel 0: ausencia de sintomatología y en la Escala del Estado Conductual de Ejecución de Karnofsky en el nivel 100 o al $100 \%$ de salud) hasta el fallecimiento del paciente, que supondría la acción total de la enfermedad sobre el organismo del paciente hasta el deterioro total (subsecuente muerte, representado en el ZUBROD en el nivel 5, y en la escala de Karnofsky en el nivel 0 o al $0 \%$ de salud).

La inferencia del estatus de salud en las escalas de ejecución conductual, parten del supuesto de que las posibilidades conductuales de un paciente oncológico, son indicativas de la conservación de salud física entre ellos. Así, tanto el ZUBROD como el Karnofsky suponen un avance progresivo de la enfermedad representado en cada nivel (numeral) conforme se asciende en la escala, para el caso del ZUBROD, o conforme se decrece en la escala, para el caso del Karnofsky. Conforme el paciente oncológico mejora o agrava en su estatus de salud física, representado en su estatus de ejecución conductual, se suma una unidad (para el caso del ZUBROD) o se restan 10 unidades o 10 puntos porcentuales (para el caso del Karnofsky) en ambas escalas respectivamente.

Estas escalas se construyeron a partir de indicadores universales de competencia inmunológica, entendida como la producción, proli- 
feración y acción eficaz de las células blancas como monocitos, leucocitos, eritrocitos y células NK (Natural Killer o asesinos naturales) presentes en el torrente sanguíneo de pacientes con cáncer. La competencia inmunológica representa (parcialmente) el estatus de salud entre estos pacientes, debido a que posibilitan el despliegue de determinados comportamientos, basados precisamente, en la fortaleza o debilidad de sus estados inmunológicos-hematológicos. La validación de este instrumento se realizó - para América Latina- por la Organización Panamericana de la Salud a inicios de la década del cincuenta, y se hizo a través del establecimiento de indicadores biológicos (marcadores biológicos) basados en la presencia de estas variables inmunológicas-hematológicas para definir los distintos estados o niveles de ejecución conductual.

\section{Cuadro 3}

Índice del Estado de Ejecución Conductual de Karnofsky

\begin{tabular}{|c|c|c|}
\hline $\begin{array}{l}\text { Estados de } \\
\text { ejecución } \\
\text { conductual }\end{array}$ & Indicadores conductuales & $\begin{array}{c}\text { Escala } \\
\text { centecimala }{ }^{a}\end{array}$ \\
\hline A & $\begin{array}{l}\text { Paciente capaz de llevar a cabo cualquier actividad en forma normal. } \\
\text { No necesita de cuidados especiales y no muestra aflicciones físicas: } \\
\text { Paciente normal; no se queja, no muestra dolencias ni evidencias de } \\
\text { enfermedad } \\
\text { Paciente capaz de culminar actividades iniciadas. Muestra señales me- } \\
\text { nores o síntomas de enfermedad } \\
\text { Paciente realiza actividades normales con esfuerzo. Evidencia señales o } \\
\text { síntomas de enfermedad }\end{array}$ & $\begin{array}{l}100 \\
90 \\
80\end{array}$ \\
\hline B & $\begin{array}{l}\text { Paciente incapaz de trabajar. Competente o en condiciones para vivir } \\
\text { en casa. Muestra aflicciones por más necesidades personales. Requiere } \\
\text { de una cantidad variable de ayuda: } \\
\text { Paciente realiza cuidados para sí mismo. Es incapaz de realizar actividades } \\
\text { normales o actividades de trabajo } \\
\text { Paciente requiere de ayuda ocasionalmente, pero es capaz de atender sus } \\
\text { propias necesidades } \\
\text { Paciente requiere de ayuda en forma considerable y cuidados médicos fre- } \\
\text { cuentes }\end{array}$ & $\begin{array}{l}70 \\
60\end{array}$ \\
\hline
\end{tabular}


Cecilia Chau Pérez-Aranibar, Hugo Morales Córdova, Micaela Wetzell Espinoza

(continuación)

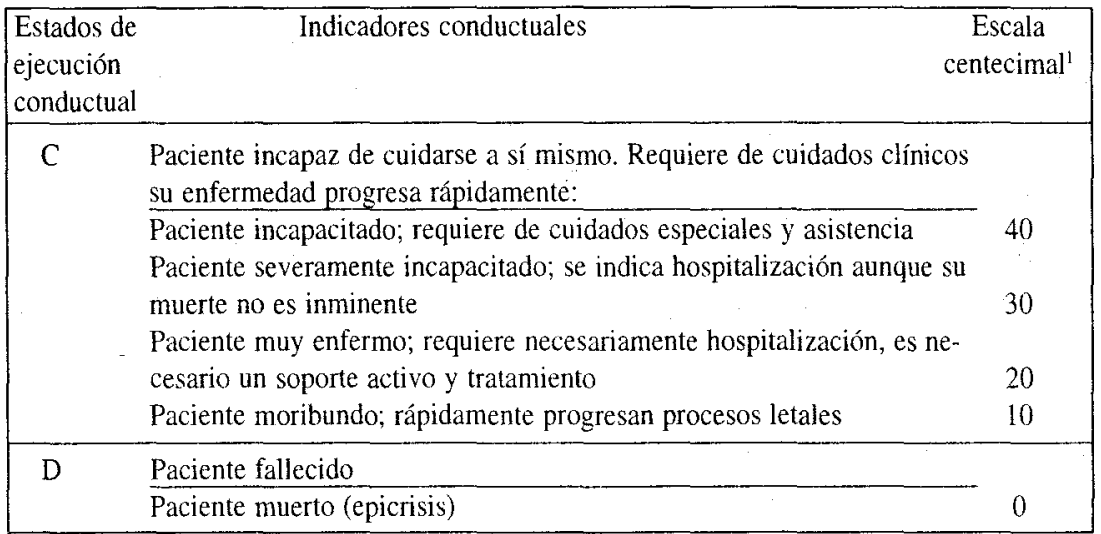

Nota. Nivel de ejectición conductual cuantificado.

Para el análisis de la significatividad estadística de los resultados alcanzada en el presente estudio -para el caso de la variable Estilos de Afrontamiento medida por el COPE- se emplearon pruebas estadísticas paramétricas y no paramétricas, luego del análisis de normalidad de distribución de las puntuaciones por escala, a través de la prueba de bondad de ajuste de Kolmogorov-Smirnov.

Entre las pruebas paramétricas empleadas, se utilizó la Prueba tStudent y ANOVA One Way para el contraste de hipótesis; y entre las no paramétricas se empleó la Prueba $\mathrm{H}$ de Kruskal-Wallis. Ambas pruebas a un nivel alfa de .05 de significatividad estadística, rechazaron la hipótesis de diferencias significativas entre grupos de pacientes por niveles de enfermedad según estilo de afrontamiento y estatus de salud, es decir, no se encontraron diferencias estadísticamente significativas entre grupos de pacientes según estilo de afrontamiento y estado de salud; aunque en un nivel descriptivo o de estimación puntual, sí se observaron diferencias importantes asociadas al uso de determinados estilos (saludables versus represivos y evitativos), así como correlaciones medianas (según el criterio de Cohen) entre dichos estilos y el estatus de salud inferido a través del estado de eje- 
cución conductual; con lo cual, afirmamos que la hipótesis de investigación se cumple en la muestra estudiada.

La validez de los resultados medidos por el COPE para este estudio, se obtuvo a través de la validez ítem-test con correlaciones inferiores a $r=.20$. La confiabilidad obtenida para el COPE en la investigación se obtuvo a través del coeficiente de consistencia interna alfa de Cronbach. Para el estilo de afrontamiento enfocado en el problema (Estilo 1): $r=.74$, para el estilo afrontamiento enfocado en la emoción (Estilo 2): $r=.66$, y para el estilo afrontamiento evitativo (Estilo $3 \mathrm{o}$ escalas adicionales propuestas por Carver, et al., 1989): $r=.55$. En todos los análisis de confiabilidad por estilos de afrontamiento, se conservó el número original de ítems en cada escala. Respecto al Índice del Estado de Ejecución Conductual de Karnofsky, se obtuvo la validez del instrumento por criterio de jueces a través de la consulta a diez jueces, todos ellos médicos oncólogos con conocimiento y práctica sobre el instrumento.

\section{Procedimiento}

Se recogieron datos relevantes para el estudio, como la medición del índice de ejecución conductual en Escala ZUBROD de las historias clínicas de cada paciente, para después proceder a registrar dichas mediciones de la Escala ZUBROD, convirtiéndolas en su equivalencia en la Escala de Karnofsky. En la primera aproximación a la muestra, se realizaron las presentaciones necesarias de los investigadores ante los pacientes, consultándoles -en forma individual y en sus habitaciones- si estaban dispuestos a participar en la investigación, informándoles de los objetivos del estudio (Anguera, 1998; Coolican, 1997; Creswell, 1994; Dyer, 1995; Heinman, 1995).

Un total de 30 pacientes de los 33 hospitalizados en el Servicio de Oncología Médica aceptaron participar. Se procedió a aplicar la Ficha de Datos Médicos y la Escala de Estimación del Afrontamiento 
COPE. El tiempo promedio de aplicación de los instrumentos para cada paciente fue de aproximadamente una hora. De los 30 pacientes evaluados, dos fallecieron, conformando finalmente 28 pacientes la muestra en estudio.

\section{Resultados}

Se escogió como media significativa un valor superior a 11 puntos, y como variabilidad significativa un valor superior a 2.91 puntos (media y variabilidad promedio, hallada entre la semisuma del valor más bajo y más alto entre los rangos) para la discusión de los resultados en el caso de las escalas, mientras que para el caso de los estilos, se optó por la semisuma de los valores 41.30 y 7.17 puntos para la media y la desviación estándar como significativas respectivamente, bajo los mismos criterios de valor máximo y mínimo entre los rangos.

De acuerdo a los resultados descritos en el Cuadro 4, se puede observar que el estilo de afrontamiento más empleado entre los participantes de la muestra, fue el enfocado en la emoción (estilo 2) con 58.75 puntos, y dentro de éste, las escalas más utilizadas fueron acudir a la religión (escala 9) con 14.21 puntos, reinterpretación positiva (escala 7) con 13.39 puntos, aceptación (escala 8) con 12.42, y apoyo social emocional (escala 6) con 11.21 puntos. El segundo estilo más empleado fue el estilo enfocado en el problema (estilo 1), con 55.64 puntos, comprendido por las escalas afrontamiento activo (escala 1) con 12.39 puntos y planificación (escala 2) con 11.82 puntos. Por otro lado, se encontró que los estilos de afrontamiento menos empleados en la muestra estudiada fueron los siguientes (reflejados en escalas): desentendimiento conductual (escala 12) con 6.46 puntos, negación (escala 10) con 7.50 puntos, enfocar y liberar emociones (escala 11) con 8.25 puntos, y supresión de actividades competentes (escala 3) con 9.50 puntos. 
Estilos de afrontamiento y estatus perfomance en un grupo de pacientes

El estilo menos empleado fue el estilo 3, conocido como Otras escalas de afrontamiento o Estilos evitativos, con 23.85 puntos. Sin embargo, de las 3 escalas correspondientes a este estilo, 2 de ellas son las que más se asocian (con correlaciones positivas y moderadas según el criterio de Cohen) al grado de enfermedad entre los participantes de la muestra estudiada; estas escalas son: enfocar y liberar emociones (escala 11, con $r=.32$ ) y desentendimiento conductual (escala 12, con $r=39$ ); más no así, la escala de desentendimiento mental, cuya correlación con la gravedad de la enfermedad es negativa y baja $(r=-.049)$.

Las escalas 5 y 4 correspondientes a la búsqueda de apoyo social por razones instrumentales y postergación del afrontamiento respectivamente, coincidieron respecto a sus puntuaciones (10.96 en cada escala); y pese a que podrían estar comprendidas entre los estilos más empleados -como comúnmente suele ocurrir- preferimos dejarlas sin incluir, dejando también de considerarlos entre los estilos menos empleados, ya que estadísticamente no están por encima del promedio de significación, sin embargo, sus puntuaciones no son tampoco, insignificantes, por el contrario, reflejan cierta presencia como los otros estilos, inclusive, entre las correlaciones con la Escala ZUBROD en su equivalencia con la Escala de Karnofsky, donde la correlación positiva es relativamente baja ( $r=.24$ y $r=.28$ respectivamente) y consistente con el modelo teórico adoptado, corroborando los supuestos y hallazgos encontrados: a mayor apoyo social instrumental, mejor estado de salud evidenciado en ejecución conductual, y a mayor postergación del afrontamiento, mayor ejecución conductual.

Sin embargo, es importante recordar que la correlación respecto a ambas variables (entre afrontamiento y salud representada en cada una de las 2 anteriores escalas) es baja según el criterio de Cohen (escala 5: $r=.24$ y escala $4: r=.28$ ). 
Cecilia Chau Pérez-Aranibar, Hugo Morales Córdova, Micaela Wetzell Espinoza

\section{Cuadro 4}

Medias y desviaciones estándar de las Escalas y Estilos de Afrontamiento del COPE $(N=28)$

\begin{tabular}{|lccccccc|}
\hline Escalas & Escala 1 & Escala 2 & Escala 3 & Escala 4 & Escala 5 & Escala 6 & Escala 7 \\
\hline $\mathrm{M}$ & 12.39 & 11.82 & 9.50 & 10.96 & 10.96 & 11.21 & 13.39 \\
$\mathrm{DE}$ & 2.13 & 2.95 & 2.57 & 2.54 & 3.70 & 3.24 & 2.20 \\
\hline
\end{tabular}

\begin{tabular}{|lcccccc|}
\hline Escalas & Escala 8 & Escala 9 & Escala 10 & Escala 11 & Escala 12 & Escala 13 \\
\hline$M$ & 12.42 & 14.21 & 7.50 & 8.25 & 6.46 & 9.14 \\
DE & 2.45 & 2.25 & 2.84 & 2.39 & 2.91 & 2.79 \\
\hline
\end{tabular}

\begin{tabular}{|lccc|}
\hline Estilos & Estilo 1 & Estilo 2 & Estilo 3 \\
\hline $\mathrm{M}$ & 55.64 & 58.75 & 23.85 \\
$\mathrm{DE}$ & 8.92 & 7.40 & 5.42 \\
\hline
\end{tabular}

De acuerdo a los resultados encontrados en las evaluaciones médico-clínicas del estado de salud, evolución de la enfermedad y pronóstico para el tratamiento (reflejados en las Escalas performance) realizadas por los oncólogos del Servicio de Oncología Médica del Hospital y registradas en las historias clínicas de los pacientes; observamos que la mitad (14 pacientes) de la muestra estudiada se ubica en el nivel 1 de Ejecución Conductual de la Escala ZUBROD, seguido de 7 pacientes ubicados en el nivel 0 , ambos grupos son pacientes -que en general- (según los estudios inmunológicos de Karnofsky) son capaces de llevar a cabo cualquier actividad en forma normal. Los del segundo grupo no necesitan de cuidados especiales y no muestran aflicciones físicas, no se quejan ni muestran dolencias ni evidencias de enfermedad, mientras que los primeros, pese a ser capaces de culminar con esfuerzo actividades comunes iniciadas, muestran señales (signos) y síntomas de enfermedad.

Por otro lado, 5 pacientes se ubican en el nivel 2. Se trata de pacientes incapaces de trabajar, pero reúnen condiciones suficientes para vivir en casa, muestran aflicciones por más necesidades personales relacionadas con su salud y requieren una cantidad variable de ayuda por problemas físicos, al igual que los 2 pacientes ubicados en 
el nivel 3, aunque estos últimos requieren de ayuda considerable y de cuidados médicos frecuentes, por lo que necesariamente tienen que estar hospitalizados. Los pacientes ubicados en el nivel 2, pese a sus deterioradas condiciones de salud, pueden aún realizar cuidados para sí mismos (como atender sus propias necesidades fisiológicas) y requieren de ayuda ocasionalmente, aunque son incapaces de realizar actividades comunes (como cocinar, barrer, etc.) y de trabajo. Aquellos pacientes ubicados en el nivel 4, no participaron en el estudio por sus graves condiciones de salud (cinco pacientes en total).

\section{Cuadro 5}

Frecuencias por nivel de Ejecución Conductual según Escala de Performance ZUBROD y su equivalencia en el Índice de Ejecución Conductual de Kamofsky (KPS)

\begin{tabular}{|c|c|c|c|c|c|c|}
\hline $\begin{array}{c}\text { Escala } \\
\text { ZUBROD }^{a}\end{array}$ & Nivel 0 & Nivel 1 & Nivel 2 & Nivel 3 & Nivel 4 & Nivel 5 \\
\hline $\begin{array}{c}\text { Frecuencia } \\
\text { de casos } \\
\text { reportados } \\
\mathrm{n}=28\end{array}$ & 7 & 14 & 5 & 2 & 0 & 0 \\
\hline $\begin{array}{c}\text { Escala } \\
\text { Karnofsky }\end{array}$ & $\begin{array}{c}\text { Estado de } \\
\text { Ejecución } \\
\text { Conductual } \\
\text { A: } \\
100\end{array}$ & $\begin{array}{c}\text { Estado de } \\
\text { Ejecución } \\
\text { Conductual } \\
\text { A: } \\
90-80\end{array}$ & $\begin{array}{c}\text { Estado de } \\
\text { Ejecución } \\
\text { Conductual } \\
\text { B: } \\
70-60\end{array}$ & $\begin{array}{c}\text { Estado de } \\
\text { Ejecución } \\
\text { Conductual } \\
\text { B / C: } \\
50-40\end{array}$ & $\begin{array}{c}\text { Estado de } \\
\text { Ejecución } \\
\text { Conductual } \\
\text { C: } \\
30-20\end{array}$ & $\begin{array}{c}\text { No existe } \\
\text { Estado de } \\
\text { Ejecución } \\
\text { Conductual } \\
\text { Paciente } \\
\text { fallecido }\end{array}$ \\
\hline
\end{tabular}

\footnotetext{
Nota. "A mayor nivel, mayor grado de enfermedad y menor estatus o nivel de salud.

${ }^{b}$ A menor puntuación, mayor enfermedad o menor nivel de salud. $N=28$
}

Con relación a las correlaciones, los valores consignados en el cuadro 6 evidencian la existencia de correlaciones medianas y positivas (según el criterio de Cohen) entre el estatus performance y las escalas 3 (supresión de actividades competentes), 4 (postergación del afrontamiento), 5 (apoyo social instrumental), 11 (enfocar y liberar emociones) y 12 (desentendimiento conductual) respectivamente. Se observa que la única correlación mediana y negativa es la escala 1 correspondiente al afrontamiento activo. 
Respecto a las primeras correlaciones por escala, se observó que a mayores niveles de enfermedad, o según la evolución o agravamiento de la enfermedad, se tiende a asociar un mayor empleo de la supresión de actividades competentes $(r=.36)$, postergación del afrontamiento $(r=.28)$, apoyo social instrumental $(r=.24)$, focalización y liberación de emociones $(r=.32)$ y desentendimiento conductual $(r=.39)$. Por el contrario, la última correlación (escala 1) sugiere que un menor empleo del afrontamiento activo se asocia a mayores niveles de enfermedad. Como se observa en el siguiente cuadro, el estilo más usado entre los pacientes -Estilo 2 enfocado en la emoción-, es el que menor correlación presenta, $r=-.04$, con el grado de enfermedad, de tal forma que se puede afirmar que a un mayor empleo del estilo de afrontamiento orientado a la emoción, se tiende a asociar un menor nivel de enfermedad en la muestra estudiada.

Respecto a las asociaciones entre la orientación del afrontamiento (hacia la emoción, el problema o la evitación) y el estatus de salud, observamos que el estilo de afrontamiento más empleado entre los pacientes de la muestra corresponde al estilo 3: otros estilos de afrontamiento o estilos evitativos, con un coeficiente de correlación de $r=.27$, seguido del estilo 1: orientado en el problema, con un coeficiente de correlación de $r=.22$. Ambas correlaciones pese a contar con coeficientes bajos, confirman la tendencia de las asociaciones típicas entre el afrontamiento y el progreso de la enfermedad entre pacientes oncológicos.

\section{Cuadro 6}

Correlaciones entre las Escalas y Estilos medidos por el COPE y los valores consignados en la Escala ZUBROD de Performance $(N=28)$

\begin{tabular}{|ccccccc|}
\hline COPE & Escala 1 & Escala 2 & Escala 3 & Escala 4 & Escala 5 & Escala 6 \\
\hline ZUBROD & -.28 & .02 & .36 & .28 & .24 & -.09 \\
\hline
\end{tabular}

\begin{tabular}{|cccccccc|}
\hline COPE & Escala 7 & Escala 8 & Escala 9 & Escala 10 & Escala 1] & Escala 12 & Escala 13 \\
\hline ZUBROD & .04 & -.00 & .04 & -.08 & .32 & .39 & -.14 \\
\hline
\end{tabular}

\begin{tabular}{|cccc|}
\hline COPE & Estilo 1 & Estilo 2 & Estilo 3 \\
\hline ZUBROD & .22 & -.04 & .27 \\
\hline
\end{tabular}


Estilos de afrontamiento y estatus perfomance en un grupo de pacientes

\section{Discusión}

Muchos de los hallazgos guardan esperada correspondencia con la literatura clásica acerca del afrontamiento entre pacientes con cáncer, mientras que otros esperan todavía mayores y mejores aproximaciones, así como plantean nuevas interrogantes y líneas de comprensión, que al parecer resultan insuficientes desde un solo modelo: el conductual-cognitivo.

Así, los resultados guardan correspondencia directa con la propuesta teórica de Lazarus y Folkman, asumida en el presente estudio, ofreciendo posibles explicaciones a los resultados encontrados. De igual forma, los planteamientos propuestos por Contrada (1990) sobre la actitud de no lucha ante determinados problemas (escala 3 ) guardan relación directa con mayores niveles de enfermedad ( $\mathrm{ZU}$ BROD) o menor estado de ejecución conductual, o menor estatus de salud (Índice Karnofsky); estas hipótesis que fueron corroboradas en el presente estudio, así como el reducido uso de la liberación y expresión de emociones como estilo de afrontamiento disposicional entre estos pacientes, el mismo que además posee una correlación moderada respecto al estatus de salud.

Sin embargo, la presencia del empleo de la escala afrontamiento activo (escala 3 ) refleja cierta inconsistencia respecto a los hallazgos y los planteamientos típicos de las teorías que explican el rol del afrontamiento disposicional antes y durante el tratamiento oncológico entre estos pacientes. Sobre esto último resulta válido preguntarnos hasta qué punto el fenómeno psicológico del afrontamiento como rasgo de la personalidad (en ese sentido disposicional, como se asume y explora en el estudio bajo el constructo estilo de afrontamiento) explica parcialmente la aparición del fenotipo del cáncer o el progreso desfavorable del mismo, frente al empleo de estrategias de afrontamiento que sugieren un uso resultante de las circunstancias específicas que experimenta el individuo, como el enfrentar esta enfermedad. 
Por lo tanto, podríamos pensar que es esperable y saludable un empleo activo del afrontamiento entre estos pacientes, considerando su estado actual de enfermedad (y hospitalización), más que pensar en una necesaria ausencia de un afrontamiento activo ante los problemas, lo cual se sugiere como característico entre estos individuos en la literatura especializada. La necesidad de construir escalas específicas de afrontamiento respecto al potencial padecimiento de esta enfermedad será un importante aporte en el conocimiento de estos pacientes y su grado de vulnerabilidad a esta enfermedad.

Así mismo, la construcción de modelos pentafactoriales que distingan las características de personalidad premórbidas al cáncer y sus estilos disposicionales de afrontamiento, versus las estrategias de afrontamiento en determinadas circunstancias (en este caso, desde el conocimiento del diagnóstico de cáncer hasta el padecimiento de sus síntomas) permitirán una comprensión efectiva de la magnitud del proceso mismo y de todas las variables que la enfermedad y su tratamiento involucran, desde el paciente, su familia y los profesionales de salud a su servicio (Cosway, Endler, Sadler y Deary, 2000).

Por otro lado, pese a que no se encontraron valores estadísticamente significativos, los hallazgos de este estudio replican la tendencia inversa de la correlación entre ambas variables, observada en los resultados reportados en el estudio de Bonadonna (1981). Este autor ha señalado que los pacientes con un mayor estilo evitativo de afrontamiento evidencian un estatus de salud más deteriorado que sus respectivos controles (pacientes con cáncer con estilos de afrontamiento no evitativos), estado característico de los participantes del estudio; mientras que los estilos de afrontamiento represivos (equivalente en el estudio a las escalas 8 y 9 , de aceptación y negación respectivamente, dentro del estilo de afrontamiento orientado a la emoción) mostraron correlaciones bajas con el grado de enfermedad y asociación inversa en el caso de la aceptación. 
Al respecto, esta aparente contradicción entre las definiciones del afrontamiento típico de pacientes oncológicos y de personalidades premórbidas al cáncer merece aún estudios posteriores que permitan la identificación definitiva de constructos unificados o factoriales que engloben de manera general la constelación de rasgos y estilos relativamente estables de enfrentar el estrés en esta población. El COPE posee escalas que presentan resultados adaptativos o desadaptativos respecto a la salud de un individuo en distintas circunstancias y en distintos momentos del ciclo vital, y pese a que existen escalas que suelen asociarse empírica y consistentemente a distintas enfermedades físicas, no se puede establecer un patrón típico de afrontamiento disposicional premórbido al cáncer únicamente desde estas escalas. Intentos en esta línea necesitarán del empleo de la validez de criterio concurrente, sea a través del diagnóstico médico o de la aparición de la enfermedad en muestras generales no sintomáticas con diseños longitudinales, y de la construcción de una escala de medición del afrontamiento con alto potencial predictivo al padecimiento de algunos tipos de cáncer (o de otras enfermedades físicas), un reto psicométrico enorme pero necesario de superar para la Psicología de la salud.

Por otro lado, los resultados de la presente investigación coinciden con los informes reportados por Cano y Vindel (1994; en Cardenal, 1997b), quienes encontraron que los pacientes con distintos tipos de cáncer (como en el presente estudio, aunque sin el control experimental deseado sobre los efectos del tratamiento y de cada tipo de cáncer en particular) comparados con un grupo control de pacientes sanos equivalentes en edad, sexo y nivel cultural, presentaban como grupo un patrón típico de alto potencial estilo evitativo o represivo de afrontamiento (el autor no conserva las distinciones entre estilo represivo y evitativo, entendiendo ambos como represión de emociones y/o evitación del distrés) asociado a un nivel bajo de competencia inmunológica y en consecuencia a un menor estatus de salud. De esta manera se corrobora la hipótesis de asociar un estilo evitativo de afrontamiento con un deficiente funcionamiento del aparato inmune 
(ocasionando daños funcionales a través del eje neuroinmunoendocrino), representado en el COPE por el estilo 3, correspondiente a los estilos evitativos de afrontamiento (especialmente a la escala 12 de desentendimiento conductual).

Respecto a este punto, es importante señalar la existencia de un problema metodológico importante en el momento de determinar la existencia de un modelo tentativo de relación causal (como parte de la multicausalidad de la etiopatogenia del cáncer) entre afrontamiento evitativo y progreso de la enfermedad, evidenciado este último en un estado de ejecución (de salud) más deteriorado o limitado, puesto que el estilo de afrontamiento podría resultar del estado específico de enfermedad y de la agresividad del tratamiento prescrito (en ese sentido, no se habría logrado una medida del afrontamiento disposicional, sino situacional), más que ser un factor potencialmente cancerígeno o que favorezca el progreso de dicha enfermedad, independientemente del tipo de cáncer y esquema terapéutico prescrito. D esta manera, parte de la personalidad del individuo constituye el factor que más explique la aparición del fenotipo maligno como la teoría lo propone.

En este sentido, el esclarecimiento de la dinámica de la enfermedad en fases iniciales e intermedias requerirá de estudios longitudinales en los que se comparen puntuaciones de un afrontamiento disposicional versus un afrontamiento situacional (como en el caso de recién conocer el diagnóstico de cáncer), en pacientes sintomáticos, donde se observe la orientación natural de varianzas significativas hacia la presencia del diagnóstico de cáncer, su progreso y el estado de salud en diferentes momentos de la enfermedad.

Al igual que para el caso de la personalidad premórbida a los trastornos cardiovasculares o patrón conductual Tipo A (PCTA), también se ha sugerido la existencia de un tipo específico de personalidad o patrón conductual que favorece o predispone al desencadenamiento de un cuadro oncológico, conocido como Personalidad Tipo 
C o patrón conductual Tipo C (Moscoso, 1994). Este constructo incluye estilos de afrontamiento disposicionales como la represión emocional, representado en el estudio en el alto empleo de la aceptación como estilo de afrontamiento enfocado en la emoción (afrontamiento fuertemente asociado a la represión emocional) y en el bajo empleo de los estilos evitativos, especialmente enfocar y liberar emociones y desentendimiento conductual. Estos últimos estilos de afrontamiento alcanzan coeficientes de correlación medianos y positivos; de este modo se evidencia la presencia del constructo PCTC a través de uno de sus componentes parciales característicos, el afrontamiento.

Así mismo, se sugiere la replicación de estudios orientados en esta línea que permitan el diseño de modelos explicativos del comportamiento de la variable afrontamiento y su asociación o concomitancia con la implementación de programas de salud de intervención directa (psicoterapia de apoyo, relajación, visualización dirigida y otras técnicas conductuales-cognitivas) que logren efectos reales -en los casos en los que sea posible- respecto a la reversibilidad, detenimiento o lentificación del progreso de las neoplasias malignas, prolongando el tiempo de sobrevida y elevando la calidad de vida de los pacientes.

En lo que respecta al rol del psicólogo de la salud, sus acciones en este campo podrían orientarse a intervenciones a dos niveles: por un lado la intervención preventiva primaria, que apuntaría a la identificación temprana de características de personalidad premórbidas al cáncer en población general no sintomática a través de la evaluación psicológica especializada, mediante entrevistas y la administración de cuestionarios de personalidad específicos con el fin de descartar la presencia de premorbidez oncológica (evaluación psiconcológica de escudriñamiento o screening); por otro lado, el desarrollo de programas de educación para la salud en los que se ofrezca información relevante sobre los efectos inmunosupresores del manejo inadecuado de variables como las emociones negativas, el pensamiento pesimis- 
ta, y los estilos de vida no saludables sobre la salud, así como la realización de talleres en los que se brinden herramientas correctivas sobre dichas variables.

En cuanto a la intervención psicológica secundaria y terciaria, se puede favorecer la competencia e incrementar la presencia de las variables inmunológicas mencionadas al ser estimuladas a través de técnicas conductuales como el condicionamiento neopavloviano, la visualización dirigida y el biofeedback, así como el uso de la psicoterapia cognitiva (por ejemplo, la terapia racional-emotiva) y la psicoterapia breve y de apoyo. Se ha evidenciado que todas ellas presentan efectos beneficiosos sobre la calidad de vida y el nivel de tolerancia farmacológica entre estos pacientes (Schneiderman, Antoni, Saab y Ironson, 2001).

Finalmente, se sugiere para la obtención de resultados y medidas de más evidente asociación entre el estilo de afrontamiento y el nivel de competencia inmunológica, correlacionar cada escala del cuestionario de afrontamiento con variables hematológicas de inmunidad, como el número de células Natural Killer, Linfocitos CD-4 y CD-8, y linfocitos T y B. Asimismo, que se lleven a cabo estudios de metaanálisis que clarifiquen y confirmen el potencial rol diferencial que juega cada una las variables asociadas a la personalidad premórbida al cáncer y a su expresión fenotípica durante el ciclo vital.

\section{Referencias}

Anguera, M. (1998). Métodos de investigación en psicología. Madrid: Síntesis.

Bleiker, E., Van-der-Ploeg, H. y Arder, H. (1995). Personality traits of women with breast cancer, before and after diagnosis. Psychological-Reports, 76 (2), 121-137.

Bonadonna, G. (1981). Dose-response effect of adjuvant chemoterapy in breast cancer. New England Journal of Medicine, 304, 10-46. 
Estilos de afrontamiento y estatus perfomance en un grupo de pacientes

Breznitz, S. y Goldberger, L. (Eds.) (1993). Handbook of stress:

Theorical and clinical aspects. Nueva York: Free Press.

Buendía, J. (1993a). Estrés y psicopatología. Madrid: Pirámide.

Buendía, J. (1993b). Estrés, desarrollo y adaptación. Madrid:

Pirámide.

Cardenal, V. (1997a). Factores psicológicos que afectan a la condición médica. En M. Ortiz-Tallo (Eds.), Trastornos psicológicos (pp. 45-68). Málaga: Aljibe.

Cardenal, V. (1997b). Variables psicosociales y su influencia en el cáncer. En M. Hombrado (Ed.), Estrés y salud (pp. 78-88). Valencia: Promolibro.

Carlson, J. y Hatfield, E. (1992). Psychology of emotion. Washington: Holt, Rinehart and Winston.

Carver, Ch. y Scheier, M. (1994). Situational coping and coping dispositions in a stressful transaction. Journal of Personality and Social Psychology, 66 (1), 184-195.

Carver, Ch., Scheier, M. y Weintraub, J. (1989). Assesing coping strategies: A theoretically based approach. Journal of Personality and Social Psychology, 56 (2), 267-283.

Cella, D. F., Orofiamma, B. y Holland, J. C. (1987). The relationship of psychological distress, extent o disease and performance status in patients with lung cancer. Cáncer, 60, 1661-1667.

Contrada, R. J., Leventhal, H. y O'Leary, A. (1990). Personality and Health. En L. Pervin (Ed.), Handbook of personality (pp. 638670). Nueva York: The Guilford Press.

Coolican, H. (1997). Métodos de investigación y estadística en psicología. México: El Manual Moderno.

Cooper, C. y Payne, R. (1991). Personality an stress: Individual differences in the stress process. Nueva York: Wiley .

Cosway, R., Endler, N., Sadler, A. y Deary, I. (2000). The coping inventory for stressful situations: Factorial structure and associations with personality traits and psychological health. Journal of Applied Biobehavioral Research, 5(2), 121-143. 
Creswell, J. (1994). Research desing: Quantitative and qualitative approaches. California: Sage.

Chau, C. (1998). Consumo de bebidas alcohólicas en estudiantes universitarios: Motivaciones y estilos de afrontamiento. Tesis para optar el grado de magíster en psicología, Pontificia Universidad Católica del Perú, Lima.

De Vita, L. (2000). Cáncer. Madrid: Interamericana.

Dyer, C. (1995). Beginning research in psychology. Oxford: Blackwell. Eysenck, H. J. (1995). The causal role of stress and personality in the etiology of cancer and coronary hearth disease. En Ch. Spielberger e I. Sarason (Eds.), Series in stress and emotion: Anxiety, anger and curiosity (pp. 3-12). Washington: Taylor \& Francis.

Flores, P. (1999). Estrés y estilos de afrontamiento en padres de niños recién diagnosticados con leucemia o linfoma noHodgkin. Tesis para optar el grado de licenciada en psicología clínica. Pontificia Universidad Católica del Perú, Lima.

Grossarth-Maticek, R. y Eysenck, H. (1990). Personality, stress and disease: Description an validation of a new inventory. Psychological-Reports, $66,355-373$.

Heinman, G. (1995). Research methods in psychology. Boston: Houghton Mifflin.

Hernández, R., Fernández, C. y Baptista, P. (1998). Metodología de la investigación. México: McGraw-Hill.

Kiecolt-Glaser, J. y Glaser, R. (1986). Psychological influences on immunity. Psychosomatics, 27 (9), 621-624.

Kiecolt-Glaser, J., McGuire, L., Robles, T. y Glaser, R. (2002). Emotions, morbidity and mortality: New perspectives from psychoneuroinmunology. Annual Reviews Psychological, 53, 83-107.

Kerlinger, F. (1988). Investigación del comportamiento. México: McGraw-Hill.

Kline, P. (1993). The handbook of psychological testing. Londres: Boutledge.

Labrador, F. y Crespo, M. (1994). Evaluación del estrés. En R. Fernández Ballesteros (Ed.), Evaluación conductual hoy: Un 
Estilos de afrontamiento y estatus perfomance en un grupo de pacientes

enfoque para el cambio de la psicología clínica y de la salud (pp. 64-89). Madrid: Pirámide.

Lazarus, R. (1991). Emotion and adaptation. Nueva York: Oxford University Press.

Lazarus, R. y Folkman, S. (1986). Estrés y procesos cognitivos. Barcelona: Martínez Roca.

Moscoso, M. (1994). La psicología de la salud: Un enfoque multidisciplinario acerca del estrés y cambio conductual. Revista de Psicología de la PUCP, 12 (1), 47-71.

Odgen, J. (1998). Health psychology: A textbook. Buckinham: Open University.

OMS (1995). Evaluación de la calidad de vida en las enfermedades crónicas. Ginebra: OMS.

Rodriguez, J. (1995). Psicología social de la salud. Madrid: Síntesis. Rojas, R. (1997). Ansiedad, cólera y estilos de afrontamiento en portadores de VIH. Tesis para optar el grado de magíster en psicología, Pontificia Universidad Católica del Perú, Lima.

Rosenzweig, M. y Leiman, A. (1993). Psicología fisiológica. Madrid: McGraw-Hill /Interamericana de España.

Schneiderman, N., Antoni, M., Saab, P. y Ironson, G. (2001). Health psychology: Psychosocial and biobehavioral aspects of chronic. Annual Reviews Psychological, 52, 555-580.

Simón, M. (1993). Psicología de la salud. Madrid: Pirámide.

Stein, M. y Miller, A. (1993). Stress, the inmune system and health and illnes. En M. Stein (Eds.), Handbook of stress: Theoretical and clinical aspects (pp. 127-315). Nueva York: Free Press.

Taylor, S. (1991). Health psychology. Nueva York: McGraw-Hill. Valdés, M. y De Flores, T. (1990). Psicobiología del estrés. Barcelona: Martínez Roca.

Valdez, N. (1999). Estrés y recursos de afrontamiento en un grupo de adolescentes embarazadas. Tesis para optar el grado de licenciada en psicología, Pontificia Universidad Católica del Perú, Lima.

Zeidner, M. y Endler, N. (Eds.) (1996). Handbook of coping: Theory, research, and applications (Eds.). Nueva York: Wiley. 